\title{
EFEITO DA AUTOCALIBRAÇÃO PARA STEREO-PIV NA DISTRIBUIÇÃO DA VELOCIDADE 2D3C DA FASE LÍQUIDA EM UMA SEÇÃO DE UMA COLUNA DE BOLHAS
}

\author{
K. K. da $\operatorname{COSTA}^{1}$; D. I. S. FORERO ${ }^{1}$; R. L. AMARAL ${ }^{1}$; O. P. TARANTO ${ }^{1}$; S.S.V. \\ VIANNA $^{1} ;$ M. MORI $^{1}$ \\ ${ }^{1}$ Universidade Estadual de Campinas, Faculdade de Engenharia Química. \\ E-mails para contato: karinakc@feq.unicamp.br,val@feq.unicamp.br, savio@ feq.unicamp.br, \\ mori@feq.unicamp.br
}

\begin{abstract}
RESUMO - A técnica PIV (Velocimetria por Imagem de Partícula), que é utilizada para determinar o campo de velocidade de diversos escoamentos, pode ser estendida para uma configuração que permite obter o terceiro componente de velocidade (2D3C), denominada Stereo-PIV. Para a utilização desta técnica é necessário relacionar as imagens obtidas com as coordenadas do espaço físico, procedimento denominado calibração, obtendo uma função de mapeamento, que contém erros. Para diminuí-los, pode-se utilizar uma técnica chamada autocalibração, que consiste em uma correção realizada após a obtenção das imagens. Esse trabalho tem como objetivo estudar o efeito da autocalibração para Stereo-PIV na distribuição da velocidade 2D3C da fase líquida em seção de uma coluna de bolhas. Foi analisada a distribuição da velocidade sem e com a autocalibração. Os ensaios experimentais foram realizados com água e ar em uma coluna cilíndrica de acrílico com altura de $1 \mathrm{~m}$ e diâmetro igual a $145 \mathrm{~mm}$.
\end{abstract}

\section{INTRODUÇÃO}

Por serem dispositivos que oferecem grandes áreas de contato entre as fases e boas características de transferência de massa e de calor, as colunas de bolhas são utilizadas como reatores multifásicos em processos que envolvem reações de fermentação, oxidação, polimerização, entre outros. Sua aplicação se estende a uma ampla gama de indústrias como química, petroquímica, biotecnológica e metalúrgica, devido a seu baixo custo de operação, simplicidade e elevada eficiência energética (Kantarci et al., 2005). As técnicas de medições por análise de imagens são de grande importância para o estudo da fluidodinâmica no interior de escoamentos multifásicos. Por se tratarem na grande maioria de sistemas não intrusivos, estas possibilitam a análise de padrões de escoamento sem a interferência do equipamento de medição no interior do equipamento. Uma destas técnicas de medição é denominada velocimetria por imagem de partícula (PIV), que permite captar imagens subsequentes e correlacioná-las a fim de obter os campos vetoriais 2D-2C ( 2 dimensões e 2 componentes) da área analisada. Por sua vez, esta pode ser estendida pela utilização de duas câmeras voltadas para a área de medição a partir de ângulos diferentes, formando o sistema denominado StereoPIV. Este consiste na análise do escoamento pela determinação da velocidade 2D-3C a partir da reconstrução da terceira componente utilizando duas projeções com ângulos diferentes 
geralmente simétricos (van Doone e Westerweel, 2007). Ou seja, no Stereo-PIV são calculados os campos 2D-2C para cada câmera e, em seguida, é realizada a reconstrução do terceiro componente.

Para a obtenção dos três componentes de velocidade com precisão, é necessário realizar um procedimento de calibração que relaciona o espaço físico com os planos de imagem por uma função de mapeamento. Uma forma de calibrar o sistema Stereo-PIV é orientar as linhas de visão das câmeras tendo como alvo uma placa plana marcada com pontos definidos distribuídos por toda sua área exatamente onde encontra-se a passagem do plano de luz laser e movimentá-la na direção $\mathrm{z}$ por uma distância conhecida, ou, como foi realizado neste trabalho, utilizar-se de duas placas planas posicionadas em sequência na direção z, com espessura definida e obter imagens de cada uma delas separadamente. As imprecisões na calibração são causadas por distorção óptica devido ao alinhamento óptico impreciso, imperfeição no design da lente, refração por janelas ópticas, interfaces de fluidos e outros elementos de óptica em um experimento (SOLOFF et al., 1997).

Além da distorção causada pela curvatura da parede da coluna (Figura 1a), a presença de bolhas no escoamento localizadas em frente ao plano investigado também interfere na qualidade das imagens obtidas por Stereo-PIV (Figura 1b). O procedimento de calibração ocorre na fase inicial, sem a alimentação de fase gasosa, logo, não possibilita a correção deste tipo de distorção. A interferência das bolhas pode ser corrigida pelo procedimento de autocalibração de Wieneke (2005), que é realizado após a gravação das imagens de partículas.

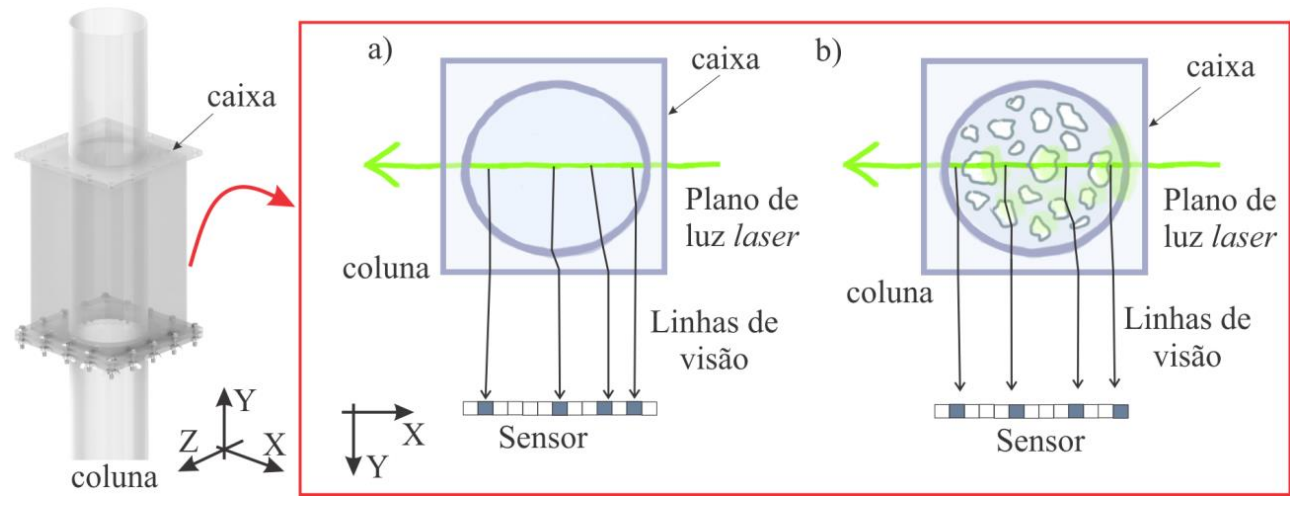

Figura 1 - Distorção causada: (a) pela curvatura do tubo e (b) pelas bolhas localizadas na frente do plano investigado.

O procedimento de autocalibração inicia após o cálculo da função de mapeamento pela observação das placas de calibração demarcadas. Idealmente, as duas imagens devem se corresponder perfeitamente. Qualquer desvio $d$ (apresentado em um mapa de disparidade) é uma indicação de algum desalinhamento conduzindo a diferentes fontes de erro (Figura 2). Os erros dos vetores de disparidade significam que as duas linhas projetadas de cada câmera não se cruzam exatamente em um único ponto. $\mathrm{O}$ ideal é encontrar um ponto cuja projeção para as duas imagens sejam o mais próximo das posições medidas (Hartley e Sturm, 1994 apud Wieneke, 2005). Em seguida, é calculado o mapa de disparidade, que foi usado por Willert (1997) para corrigir a posição na qual os vectores $2 \mathrm{C}$ correspondentes são calculados para as câmaras 1 e 2 . Uma vez que o mapa de disparidade tenha sido computado, um ponto real no plano de medição é computado por um método de triangulação padrão para cada vetor (Figura 2b). Em seguida, os coeficientes da função de mapeamento de imagem são ajustados para 
corrigir o desfasamento entre o plano de calibração e o plano de medição. Consequentemente, a disparidade da imagem é absorvida como uma deformação adicional na função de mapeamento do sistema (Wineke, 2005). O procedimento também pode ser aplicado em casos onde há diferenças substanciais entre a placa de calibração e a área de medição. Tornando-se assim, uma solução para os sistemas fechados onde não é possível inserir a placa de calibração no interior do sistema (Adrian e Westerweel, 2011). Por fim, o plano é ajustado na folha de luz e uma nova função de mapeamento é calculada sendo este plano definido como z $=0 \mathrm{~mm}$ e são definidos o novo ponto de origem xy e a direção do eixo x. Um fluxograma geral do procedimento de autocalibração é demonstrado na Figura $2 \mathrm{a}$.
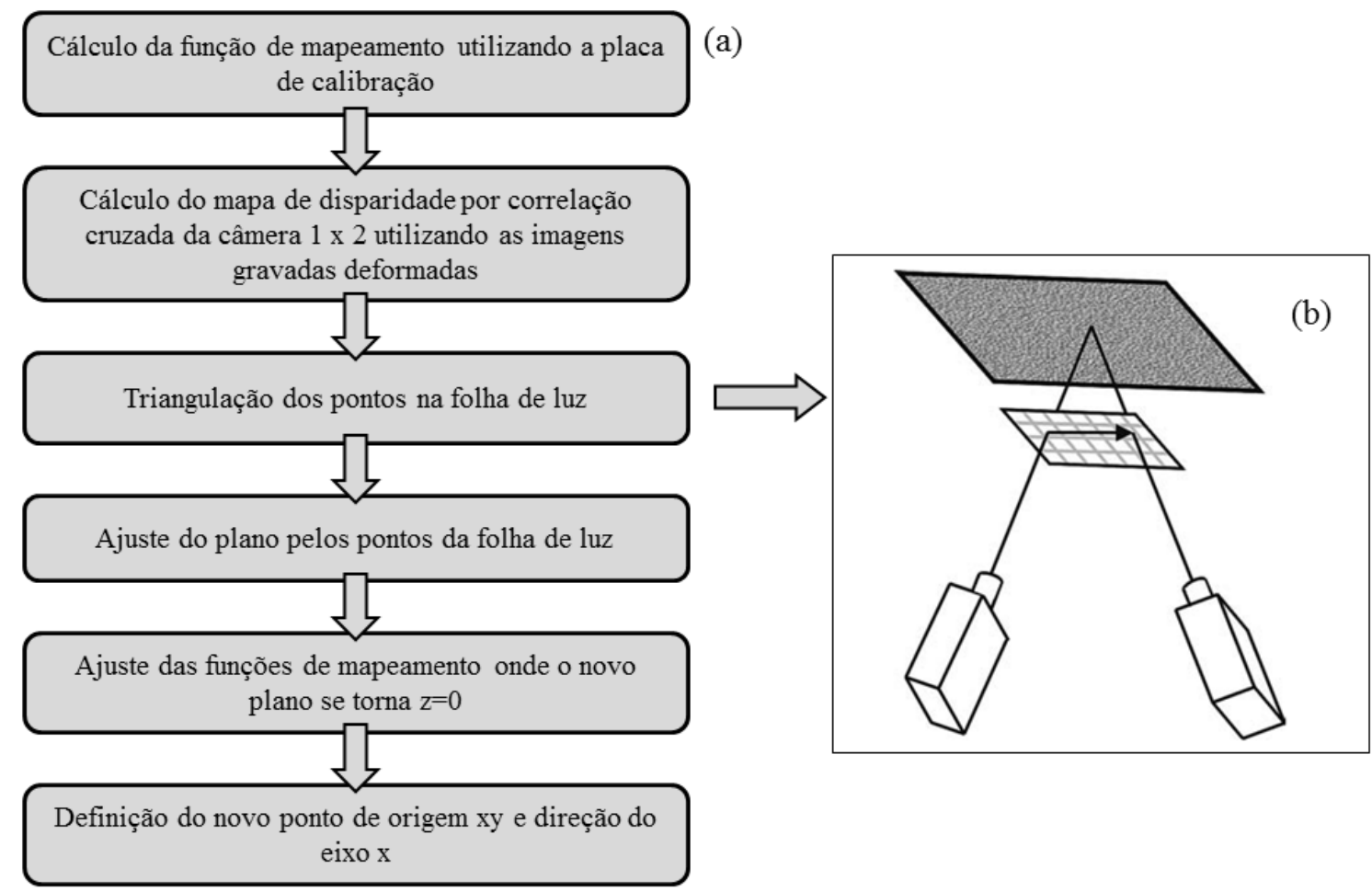

Figura 2 - (a) Fluxograma do procedimento de autocalibração; (b) os pontos reais da folha de luz podem ser compuados por triangulação utilizando oos vetores de disparidade (adaptado de Wieneke (2005)).

O objetivo deste trabalho é então, avaliar a influência do procedimento de autocalibração na distribuição de velocidade 2D-3C da fase líquida de uma coluna de bolhas.

\section{PROCEDIMENTO EXPERIMENTAL}

No procedimento experimental, fez-se o uso de uma coluna de bolhas cilíndrica construída em acrílico, com diâmetro de $14,5 \mathrm{~cm}$ e altura de $100 \mathrm{~cm}$ (Figura 3a). A mesma foi preenchida com água a $25^{\circ} \mathrm{C}$ com altura inicial da fase líquida de $70 \mathrm{~cm}$ e a fase gasosa consiste em ar na mesma temperatura adentrando no sistema pela parte inferior, passando por um distribuidor de placa plana de 21 furos igualmente distribuídos em um arranjo quadrado central (Figura 3c). A vazão de gás foi fixa em $2 \mathrm{~L} / \mathrm{min}$. A altura central da área de medição está localizada a 55,2 cm do distribuidor e consiste em 14,5 x $13 \mathrm{~cm}$ (Figura 3b). O sistema de 
aquisição de imagens da Stereo-PIV é formado por duas câmeras CCD (Charge Couple Device) com lentes objetiva com distância focal de $60 \mathrm{~mm}$ modelo Nikon Micro-Nikorr (f\#2.8D) e um sistema laser Nd:YAG (200 mJ/pulso e $\lambda=532 \mathrm{~nm}$ ) (Figura 3d) sistema controlado por uma unidade sincronizadora interna (PTU-9) pelo software Davis 7.2, fornecido pela empresa LaVision. Como material traçador, necessário para calcular o campo de velocidade da fase líquida, fez-se o uso de Rodamina B, material fluorescente que emite um comprimento de onda $\lambda=620 \mathrm{~nm}$ quando excitado pela luz laser. Acoplados às câmeras, foram utilizados filtros passa-alta, que restringem a passagem de luz no comprimento de onda do traçador, de forma a evitar a passagem de reflexões e demais ruídos que prejudicam a imagem obtida e podem danificar o equipamento. Foram gravadas 1000 imagem em uma frequência de $4,92 \mathrm{~Hz}$. A espessura do feixe é definida pela presença de uma caixa com fenda de espessura conhecida que restringe a passagem da luz (Figura 3a, b).



Figura 3 - Visão geral do sistema experimental usado neste trabalho: (a) coluna de bolhas, sistema laser, caixa com fenda e posicionamento das câmeras CCDs; (b) área de investigação e definição dos eixos x, y e z; (c) detalhe do distribuidor de 21 furos; (d) visão superior do arranjo do sistema Stereo-PIV na coluna.

De acordo com Adrian e Westerweel (2011), há vários arranjos possíveis para o posicionamento das câmeras em relação ao plano de luz laser. O arranjo utilizado neste trabalho possui ambas as câmeras posicionadas em frente ao plano de luz laser com um ângulo entre elas de $60^{\circ}$ mantendo a simetria em relação à área investigada (Figura 3a). $\mathrm{O}$ posicionamento das câmeras no mesmo lado é favorável, pois o acesso óptico é requerido 
somente em uma direção. O plano das câmeras é perpendicular ao plano da área investigada (Figura 3d), e a iluminação gravada pelas câmeras é aproximadamente a mesma já que a condição de simetria existe. Na calibração, foi utilizada uma função polinomial de terceira ordem como função de mapeamento com um erro médio de 0,6619 pixel. Os erros para os planos $\mathrm{z}=-4 \mathrm{~mm}$ e $\mathrm{z}=0$, para a câmera 1 foram de 0,5924 e 0,7002 pixel, respectivamente e para a câmera 2, 0,5767 e 0,7783 pixel.

O tempo entre o frame 0 e 1 (dt) utilizado foi de $2000 \mu$ s, a potência do laser de $35 \%$ e a espessura da folha de luz laser é de $4 \mathrm{~mm}$, coincidente com o posicionamento das placas de calibração. Foi aplicado um pré-processamento nas imagens obtidas utilizando o filtro RMS (Roots Mean Square) 3x3 pixels e no processamento dos campos vetoriais foi utilizado uma estratégia de interrogação com dois passos, sendo o primeiro e o segundo com um tamanho de 128 pixels e 64 pixels, respectivamente. Para a análise do efeito do procedimento de autocalibração, três parâmetros foram variados, dx, dy e overlap, e os campos vetoriais foram processados com cada função de mapeamento corrigida. Os parâmetros dx e dy representam o deslocamento da imagem nos eixos $\mathrm{x}$ e $\mathrm{y}$, respectivamente, e overlap representa a porcentagem da janela de interrogação sobreposta na vizinhança. O número de passos foi fixado em 1 e o tamanho da janela de interrogação de 64 pixels. Foi analisada a diferença entre os campos vetoriais em função do erro de triangulação resultante igual a 27, 8, 7 e 6 pixels.

\section{RESULTADOS E DISCUSSÕES}

Para avaliar o procedimento de autocalibração, foi utilizado como principal resultado o erro de triangulação, que de acordo com as especificações da LaVision (2005), deve ser menor que 5-10 pixels (Tabela 1). O desvio médio do novo polinômio de terceira ordem calculado também pode ser utilizado para avaliar a validade do novo modelo, sendo que se < 1 pixel possui um ajuste muito bom, $<2-3$ pixels resultado adequado e em caso de $>10$ pixels, o resultado é questionável.

A Tabela 1 apresenta o erro da nova função de mapeamento em função do erro de triangulação detalhando os valores de $\mathrm{dx}$, dy e overlap escolhido. A Tabela 2 apresenta os parâmetros de deformação da imagem após o procedimento de autocalibração. Percebe-se que o experimento 3 e 5 apresentaram o menor erro da nova função de mapeamento com uma média de 0,0375 e 0,0619 pixel, respectivamente. Mesmo com um erro de triangulação de 6 pixels, os experimentos 1 e 2 apresentaram uma disparidade média computada alta $(21,3106$ e $17,0737 \mathrm{~mm})$, em relação aos outros experimentos.

Tabela 1 - Parâmetros de autocalibração: erro da nova função de mapeamento em função do erro de triangulação.

\begin{tabular}{|c|c|c|c|c|c|c|c|c|}
\hline Exp & $\mathrm{dx}$ [pixel] & $\begin{array}{c}\mathrm{dy} \\
{[\text { pixels] }}\end{array}$ & $\begin{array}{c}\text { Overlap } \\
{[\%]}\end{array}$ & $\begin{array}{c}\text { Erro de } \\
\text { triangulação }\end{array}$ & \multicolumn{2}{|c|}{ Erro (Câmera 1) [pixel] } & \multicolumn{2}{|c|}{ Erro (Câmera 2) [pixel] } \\
\cline { 6 - 10 } & & & $\mathrm{z}=-4 \mathrm{~mm}$ & $\mathrm{z}=0 \mathrm{~mm}$ & $\mathrm{z}=-4 \mathrm{~mm}$ & $\mathrm{z}=0 \mathrm{~mm}$ \\
\hline 1 & 2 & -200 & 15 & 6 & 0.1374 & 0.1374 & 0.1345 & 0.1345 \\
\hline 2 & 2 & -160 & 0 & 6 & 0,1423 & 0,1423 & 0,1395 & 0,1395 \\
\hline 3 & 2 & -50 & 0 & 7 & 0,0379 & 0,0379 & 0,0371 & 0,0371 \\
\hline 4 & 2 & -195 & 15 & 8 & 0,0988 & 0,0988 & 0,0968 & 0,0968 \\
\hline 5 & 0 & 0 & 0 & 27 & 0,0626 & 0,0626 & 0,0611 & 0,0611 \\
\hline
\end{tabular}


Tabela 2 - Parâmetros de deformação da imagem após o procedimento de autocalibração.

\begin{tabular}{|c|c|c|c|c|c|c|}
\hline \multirow{2}{*}{ Exp. } & \multirow{2}{*}{$\begin{array}{c}\text { Disparidade média } \\
\text { computada [mm] }\end{array}$} & $\begin{array}{c}\text { Quantidade de vetores } \\
\text { excluídos [\%] }\end{array}$ & \multicolumn{2}{|c|}{$\begin{array}{c}\text { Rotações nos } \\
\left.\text { eixos [ }{ }^{\circ}\right]\end{array}$} & \multirow{2}{*}{$\begin{array}{c}\text { Translação no } \\
\text { eixo z (mm) }\end{array}$} & $\begin{array}{c}\text { Desvio médio } \\
\text { do plano (pixel) }\end{array}$ \\
\cline { 4 - 5 } & 21,3106 & 28 & $-0,1661$ & $-1,1023$ & 17,2138 & 6,0954 \\
\hline 1 & 17,0737 & 29 & $-0,4366$ & $-1,1114$ & 13,751 & 4,9865 \\
\hline 3 & 5,55418 & 28 & $-0,2937$ & $-0,1641$ & 4,5467 & 6,2680 \\
\hline 4 & 20.8648 & 23 & $-0,1495$ & $-0,7920$ & 16,8423 & 5,4926 \\
\hline 5 & 2,78791 & 6 & 0,0760 & $-0,4832$ & 0,7231 & 7,1355 \\
\hline
\end{tabular}

\subsection{Campos vetoriais Vx e Vy}

Uma forma de analisar o escoamento na coluna de bolhas é pelo uso de campos vetoriais médios e instantâneos dos componentes de velocidade. Por ser mais sensível aos erros da função de mapeamento, foi usada a terceira componente da velocidade $\mathrm{Vz}$ para avaliar o efeito dos parâmetros no campo vetorial. $\mathrm{O}$ valor de $\mathrm{Vz}$ varia na área investigada decorrente à ascenção das bolhas na coluna, isto pode ser percebido na Figura 4a,que apresenta um campo de velocidade instantâneo do escoamento. Na seção da coluna investigada, a distribuição da média de Vz também representa a influência da fase gasosa sobre a líquida (Figura 4b).

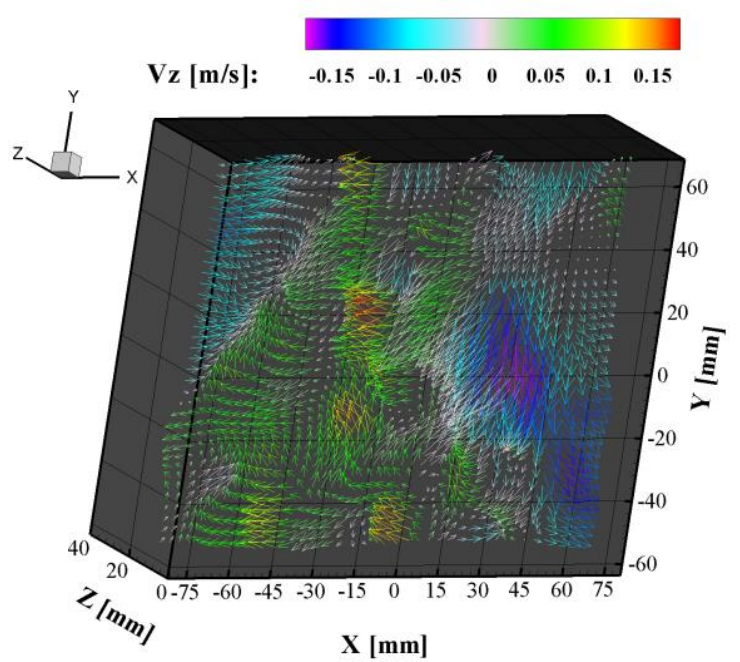

(a)

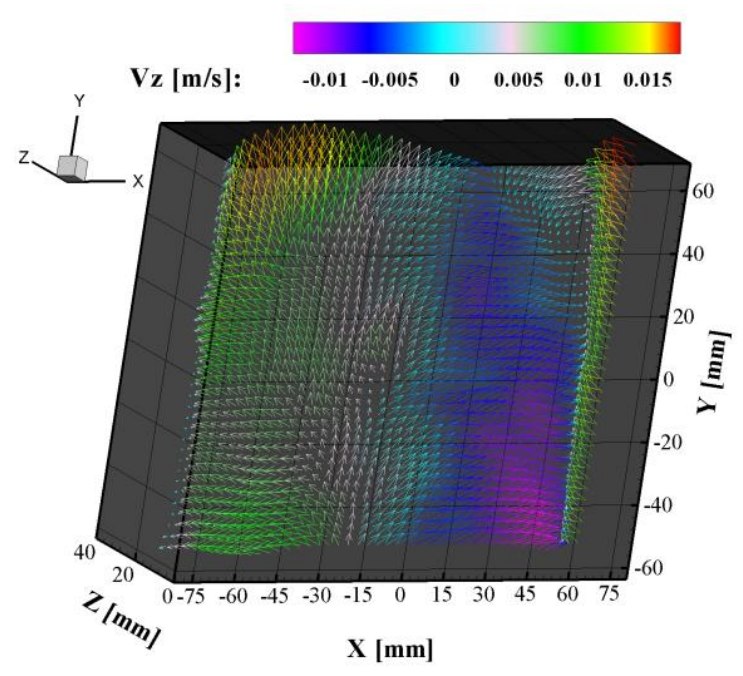

(b)

Figura 4 - Distribuição (a) instantânea e (b) média de Vz sem a autocalibração.

$\mathrm{Na}$ Figura 5 são apresentados os vetores de velocidade $\mathrm{Vx}$ e $\mathrm{Vz}$ com destaque para o campo vetorial Vy intantâneo (imagem 500) e médio. No campo vetorial médio (Figura 5b) pode-se observar o perfil de Vy típico em colunas de bolhas, com velocidades ascendentes elevadas na região central e velocidades negativas próximo às paredes da coluna. 


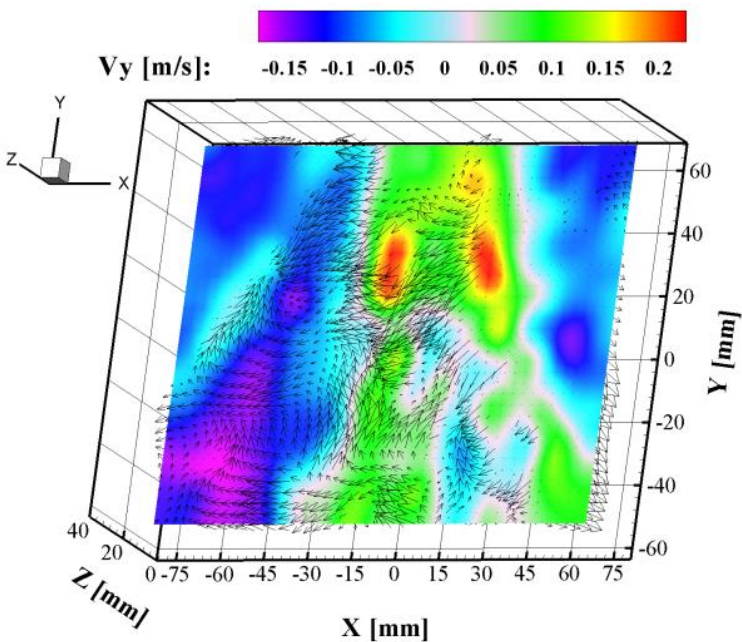

(a)

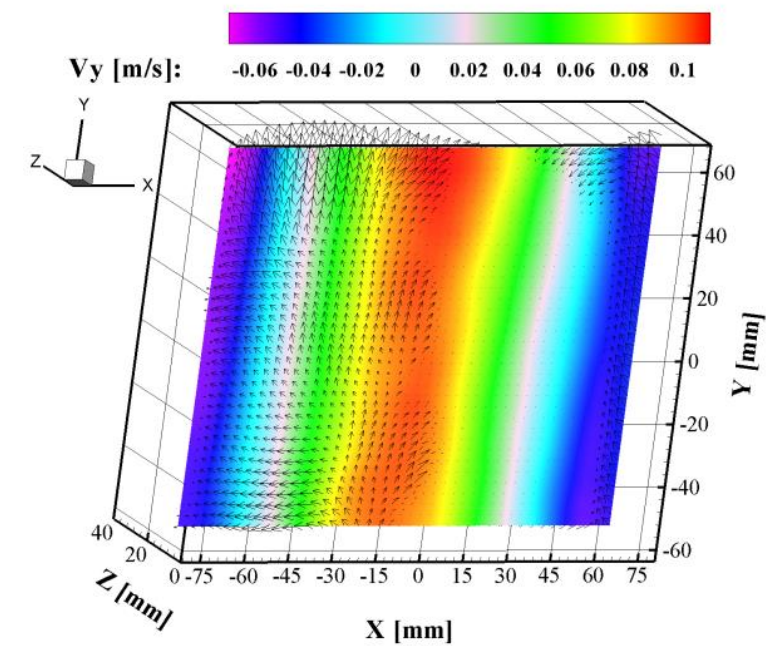

(b)

Figura 5 - Campos de velocidade 2D-3C (a) instantâneo e (b) médio.

A Figura 6 apresenta a função densidade de probabilidade não normalizada para $\mathrm{Vz}$ instantâneo e médio para cada experimento de autocalibração. Percebe-se que há variação da distribuição de $\mathrm{Vz}$ instantâneo e médio com os diferentes procedimentos de autocalibração apresentados na Tabela 2 e 3.

A Figura 6b é o histograma para a média das 1000 imagens que reforça o perfil obtido na Figura $4 b$ onde a maior parte dos vetores são positivos na direção z. Já a Figura $6 a$ permite afirmar que a aplicação da autocalibração nas imagens obtidas reduz consideravelmente a quantidade de pontos onde $\mathrm{Vz}=0$ neste instantâneo. Apesar do escoamento ser preferencialmente na direção $\mathrm{y}$, a passagem da bolha pela coluna gera regiões de circulações locais da fase líquida imediatamente após a bolha, e, por consequência, gradientes de velocidade $\mathrm{Vz}=0$, o que indica que o procedimento de autocalibração permite a melhor visualização deste componente de velocidade.

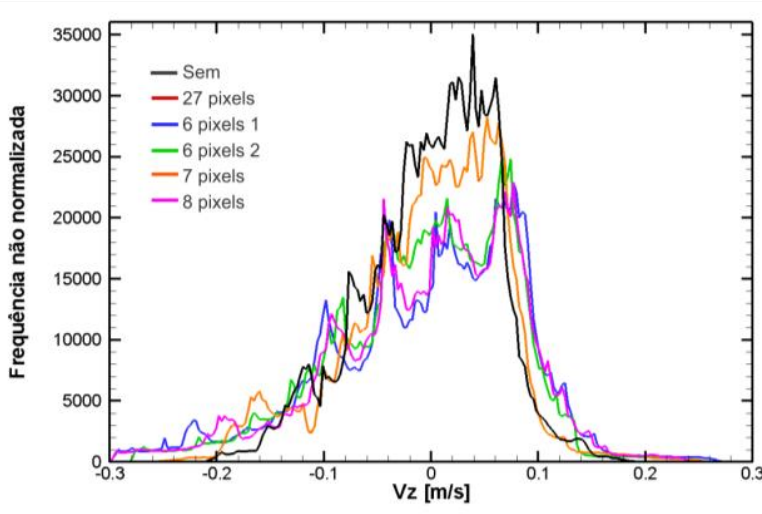

(a)

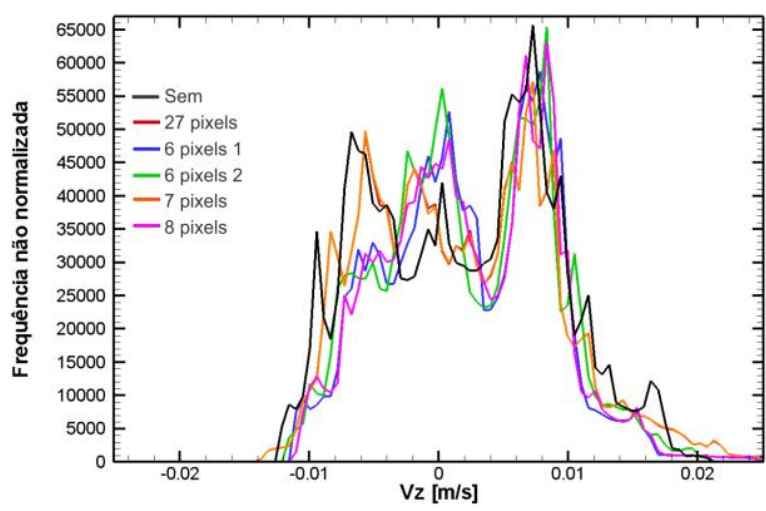

(b)

Figura 6 - Função densidade de probabilidade não normalizada para Vz (a) Instantâneo e (b) médio para cada experimento de autocalibração. 


\section{CONCLUSÕES}

As técnicas de visualização de escoamento representam uma ferramenta muito útil no estudo da fluidodinâmica de escoamentos multifásicos. No caso de Stereo-PIV, é importante relacionar as imagens obtidas com o volume físico a ser mensurado de forma criteriosa. Para isto, o procedimento de autocalibração permitiu aprimorar as medições realizadas com valores adequados aos padrões de escoamento esperado para coluna de bolhas, especialmente na velocidade Vz.

\section{AGRADECIMENTOS}

Os autores agradecem ao apoio financeiro da Petróleo Brasileiro S. A. - PETROBRÁS, termo de compromisso 0050.0070828.11.9, que garantiu o desenvolvimento deste trabalho e à CAPES.

\section{REFERÊNCIAS}

ADRIAN, R.J.; WESTWERWEEL, J. Particle Image Velocimetry. Nova York: Cambridge University Press, 2011.

KANTARCI, N.; BORAK, F.; ULGEN, K.O. Bubble Column Reactors. Process Biochemestry, v. 40, p. 2263-2283, 2005.

VAN DOONE, C. W. H.; WESTERWEEL, J. Measurement of laminar, transitional and turbulent pipe flow using Stereoscopic-PIV. Exp. Fluids, v. 42, p. 259-279, 2007

WIENEKE, B. Stereo-PIV using self-calibration on particle images. Exp. Fluids, v. 39, p. 267-280, 2005.

LAVISION. Product-Manual: FlowMaster. Göttingen. 2005.

SOLOFF, S.; ADRIAN, R.; LIU, Z. Distortion compensation for generalized stereoscopic particle image velocimetry. Measurement Science and Technology, v. 8, p. 1441-1454, 1997.

WILLERT, C. Stereoscopic digital particle image velocimetry for application in wind tunnel flows. Measurement science and technology, v. 8, p. 1465-1479, 1997. 